\title{
Optical chromatography. A new tool for separation of particles
}

\author{
T. Imasaka
}

Department of Chemical Science and Technology, Faculty of Engineering, Kyushu University, Hakozaki, Fukuoka 812, Japan

\begin{abstract}
A laser beam is focused into the solution containing particles counter-flowing in a capillary. The particle is focused into the beam center and drifts at which point the radiation pressure is identical to the force induced by the liquid flow, resulting in separation of particles as a function of size.
\end{abstract}

n 1906 Tswett reported on a separation technique which is now called chromatography [1]. This classical chromatography involves the adsorption (or extraction) of a substance to the stationary phase. The analyte can then be eluted from the stationary phase by passing a solvent, which desorbs the analyte from the support, thereby affecting a separation. Chromatography has been studied and widely used by many researchers. In practical analysis, chromatography is frequently employed for purification of the sample and for separation of the components before spectrometric determination. Chromatography, however, has several limitations that have not yet been overcome.

In 1970, Ashkin reported an optical trapping technique [2]. Under the radiation pressure, a particle with a higher refractive index than the medium is trapped at the focal point. This technique allows the manipulation of a single particle by complete holding like "adsorption" or "extraction". Recently, a new and potentially useful analytical method has been reported for the separation of particles (or molecules) using radiation pressure by incomplete holding under a liquid flow. The overall technique affects a chromatographic-like separation, but involves principles that are completely different from classical chromatography. Thus this technique is called "optical chromatography". The approach is very simple; a laser beam is focused into the solution containing substances (e.g., particles) counter-flowing in a capillary. The particle drifts at which point the radiation pressure is equilibrated with the force induced by the liquid flow, resulting in separation of particles.

In this review article, the basic principle and the analytical applications such as separations of polymer beads, yeasts, human erythrocytes are reported. This analytical method is also applied to the determination of protein based on immunoassay and even in measurements of the microorganism's power.

\section{Experimental}

A typical analytical instrument for optical chromatography is shown in figure 1 [3]. The laser beam, e.g., an argon ion

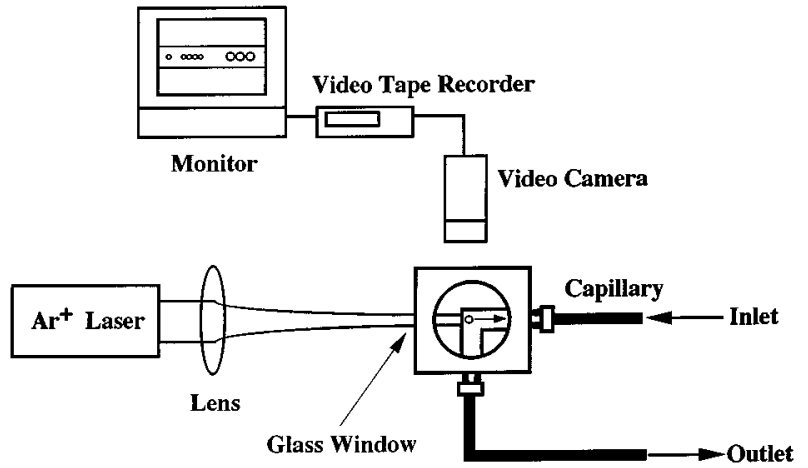

Figure 1. Block diagram of the experimental apparatus.

laser $(488 \mathrm{~nm}, 500 \mathrm{~mW})$, is focused into a capillary which is inserted into a quartz cell. The ends of the capillaries are immersed into reservoirs containing buffer solutions. The sample is introduced by gravity using a siphon method. A typical flow rate is $100 \mu \mathrm{m} \mathrm{s}^{-1}$, which is adjusted by changing the levels of the buffer solution. The motion of the particles is observed by a video camera equipped with a microscope objective. The visual data are stored in a video tape recorder.

\section{Results and discussion}

\section{Demonstration of particle separation}

Under radiation pressure, three forces are applied to the particle flowing in a medium, as shown in figure 2 . The gradient force attracts the particle into the center line of the beam, and the scattering force accelerates the particle in the direction of the incident beam. On the other hand, the medium flow pushes the microspheres downstream. Thus, the particle is trapped at the position where the scattering force is identical to the force induced by the medium flow. The motion of the particle under radiation pressure is schematically shown in figure 3. (A) a particle introduced in a capillary is focused into the center line of the laser beam by the gradient force and is turned around by the scattering force. (B) the particle is accelerated and decelerated before and after the beam waist, respectively. (C) the particles drift when the radiation pressure becomes identical to the force induced by the liquid flow. The $1-\mu \mathrm{m}, 3-\mu \mathrm{m}$, and $6-\mu \mathrm{m}$ particles are clearly separated each other by this technique [4]. Similarly, single and coagulated yeast cells are readily be 
separated. This technique is also applied to the separation of human erythrocyte cells [3]. Two fractions, one consisting of cells ranging from 1.5 to $2.4 \mu \mathrm{m}$ in diameter and another consisting of cells ranging from 3.5 to $5.7 \mu \mathrm{m}$ in diameter, are observed. These results clearly indicate that particles can be separated using this technique as a function of particle size.

\section{Parameters affecting separation}

The parameters affecting the separation of particles are theoretically calculated, based on the ray-optic model $[5,6]$. For example, the distance of the particle from the beam waist is calculated, which is shown in figure 4 [3]. In the middle of the range, the distance from the beam waist is proportional to the square root of the particle diameter, which is ascribed to the facts that the scattering force is proportional to the square of the particle diameter (or to the area receiving the light) and that the resistance force against the medium flow is proportional to the particle diameter. The particles with smaller diameters flow downstream and are not trapped, since the laser beam cannot be infinitely focused at the beam waist. The particles with large diameters also flow downstream, since the radiation force that a particle receive is limited, when the particle diameter is larger than the beam diameter. The parameters corresponding to dynamic range, selectivity, theoretical plate number, and resolution in conventional chromatography are also calculated. It is possible, in theory, to distinguish particles whose diameters differ by less than $1 \%$ for particles with a diameter larger than $1 \mu \mathrm{m}$.

\section{Application to immunoassay}

A protein molecule is frequently analyzed by immunoassay. The principle of this technique based on optical chromatography is shown in figure 5 [7]. The solution containing the protein (antigen) is mixed with polystyrene beads (immunobeads) coated with an antibody which are coagulated via an immunological reaction. The mixture of bound (B) and free (F) beads is separated by optical chromatography. Thus real-time $\mathrm{B} / \mathrm{F}$ separation is accomplished. The immunobeads drifting at the equilibrated position are combined each other in the presence of antigen by immunological reaction. This binding event can be visually observed by monitoring the motion of the coagulated bead toward its new position of equilibrium. The time for binding is reciprocally proportional to the concentration of the antigen molecule, and then an analytical curve can readily be constructed. Dissociation of the coagulated bead can also be confirmed by monitoring the motion of the combined bead, after separation, toward the original position. The time for dissociation provides us information on the rate constant of the dissociation in the immunological reaction. Thus the rates of the forward and reverse immunological reactions can be independently determined by measuring the times of formation and dissociation, respectively, of the immunobeads. Experimentally, neither distribution of the dissociation time nor dependence of antigen concentration was observed within the experimental error. This strongly suggests that the

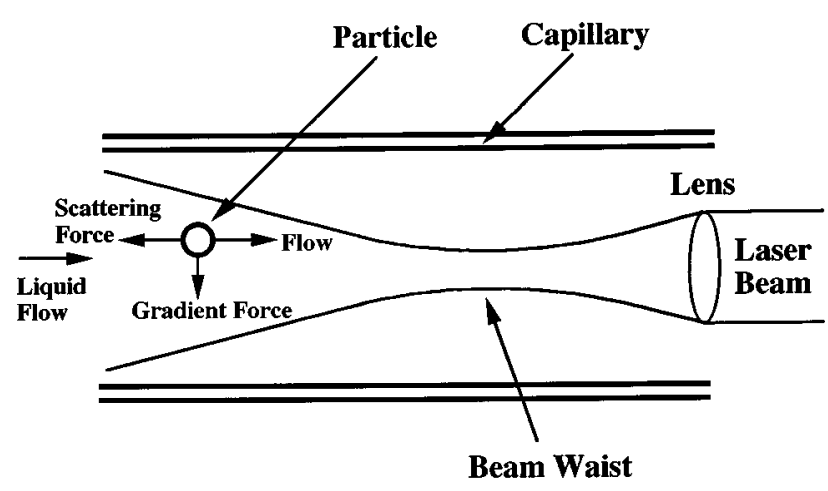

Figure 2. Forces applied to the particle flowing in a medium.
A

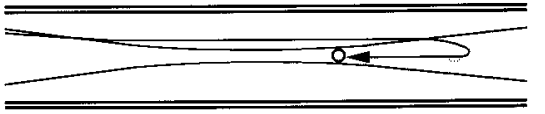

Trap and

Turn Around

$\mathbf{B}$

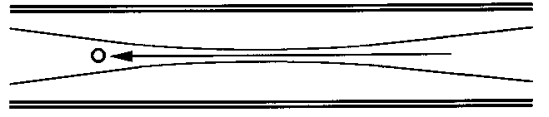

Acceleration and

Deceleration

C

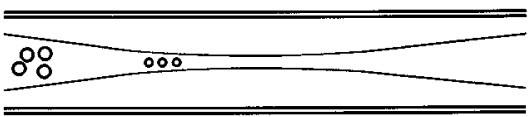

Equilibrium
Figure 3. Schematic motion of particle. The laser beam is introduced from the right-hand side and the liquid from the left-hand side.

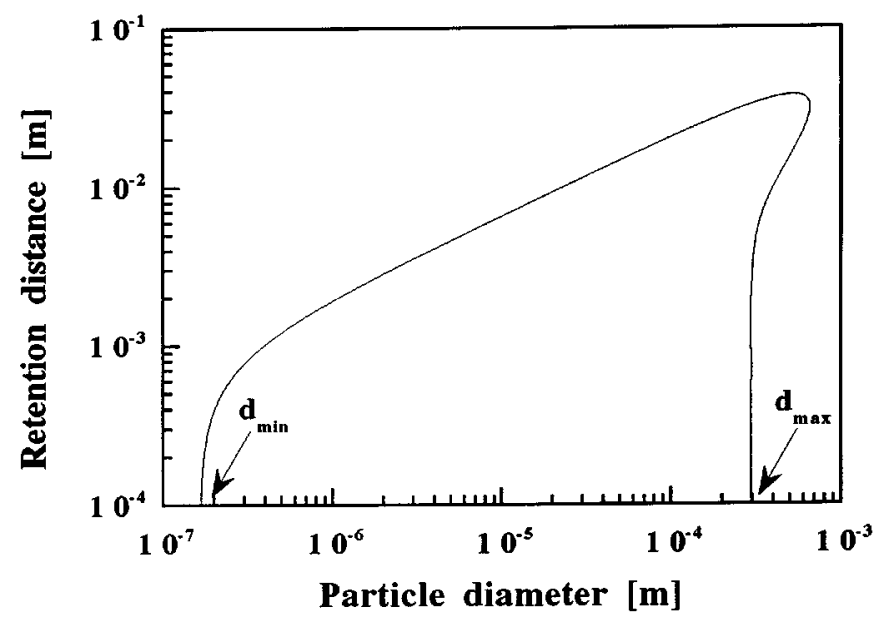

Figure 4. Relationship between retention distance and particle diameter. The retention distance is calculated using the following parameters. Laser power, $1 \mathrm{~W}$; wavelength, $488 \mathrm{~nm}$; refractive index of particle, 1.59; beam waist size, $10 \mathrm{~mm}$; flow rate, $50 \mathrm{~mm} \mathrm{~s}^{-1}$; viscosity, $8.9 \times 10^{-4} \mathrm{~Pa} \mathrm{~s}$. 
(A)

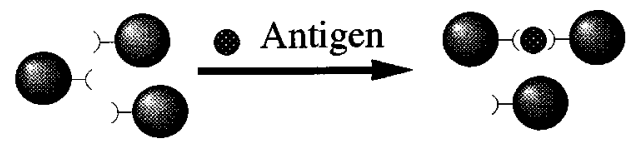

(B)

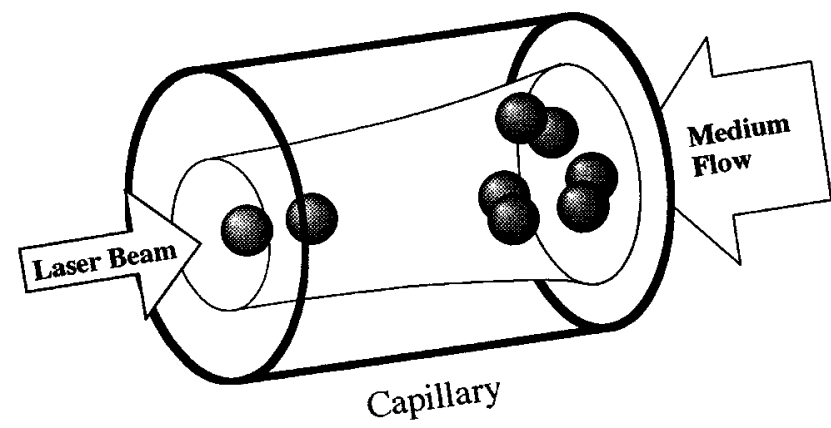

Figure 5. The concept of immunoassay using optical chromatography. (A) formation of a pair of beads by agglutination (B) B/F separation by optical chromatography.

immunobeads are combined at a single site via a single chemical bond. Thus it might be possible, in theory, to detect a single protein molecule at very low concentrations by the present approach, since a single coagulated particle can easily be separated and measured by optical chromatography.

\section{Measurement of microorganism's power}

Under equilibrium of the laser radiation force and the liquid flow, a microorganism is attracted into the beam center by a gradient force. The microorganism escapes against the trapped force which is calculated from the position where the microorganism moves away. This scheme allows the calculation of the average power for an organism with a minimum of effort, in terms of repetitive samplings and measurements. A trachelomonas volvocina is determined to have a power of c.a. $1 \mathrm{pN}[8,9]$.

\section{Comparison with other methods}

There are several approaches for separation of the particles and for determination of the particle size, e.g., field-flow fractionation and flow cytometry. However, optical chromatography has several advantages over other separation methods with respect to sensitivity, resolution, and time for separation and detection. For example, the collection efficiency can, in theory, be improved to $100 \%$ by increasing the laser output power. The separation resolution, which is mainly affected by the stabilities of the laser and liquid-flow parameters, can be improved by optimizing conditions, though a Brownian motion of the particle is an unavoidable limiting factor. Moreover, concentration and separation can be performed simultaneously, and then the present method can be applied to diluted samples. However, optical chromatography has also disadvantages. It cannot be applied to small particles and molecules, since a high-power laser emitting at the ultraviolet is required. Furthermore, the number of the particles measured in optical chromatography is limited, generally decreasing the accuracy in the measurement. Due to differences in operation principle, optical chromatography may have its own application fields, such as single molecule detection and characterization, e.g., measurements of the immunological reaction rates, or measurements of the microorganism's (e.g., sperm's) power. Such applications will be employed in various research fields such as in biological and life sciences in the future.

\section{References}

1. Pecsok, R. L.; Shields, L. D., Cairns, T.; McWilliam, I. G. Modern Methods of Chemical Analysis (2nd ed.); John Wiley \& Sons, New York, 1968.

2. Ashkin, A. Phys. Rev. Lett. 1970, 24, 156.

3. Kaneta, T.; Ishidzu, Y.; Mishima, N.; Imasaka, T. Anal. Chem. 1997, 69, 2701.

4. Imasaka, T.; Kawabata, Y.; Kaneta, T.; Ishidzu, Y. Anal. Chem. 1995, 67, 1763.

5. Ashkin, A. Biophys. J. 1992, 61, 569.

6. Roosen, G.; Imbert, C. Phys. Lett. 1976, 59A, 6.

7. Hatano, T; Kaneta, T.; Imasaka, T. Anal. Chem. 1997, 69, 2711.

8. Imasaka, T. Proc. 18th Int. Symp. Capillary Chromatogr. 1996, 1,33 .

9. Mishima, N.; Kaneta, T.; Imasaka, T. Anal. Chem., in press, 1998. 